\title{
Matching is the Key Factor to Improve the Production of Patchoulol in the Plant Chassis of Marchantia paleacea
}

\author{
Yaojie Zhang, Lu Zhou, Kexuan Tang, Meihui Xu, and Zhiqi Miao* \\ Cite This: ACS Omega 2020, 5, 33028-33038 \\ Read Online
}

ABSTRACT: The valuable terpenoids, such as artemisinin acid, have achieved bioproduction in the chassis of microbes recently. In this study, Marchantia paleacea L, a promising plant synthetic biology chassis, was used to explore the possibility of patchoulol production by constructing a synthetic biology pathway composed of FPS and PTS. The experiment results show that the maximum yields based on the cytoplasm and plastid pathway were 621.56 and $1006.45 \mu \mathrm{g} / \mathrm{g}$, respectively. However, there is no statistically significant difference in the yield of patchoulol between transformant plants with different subcellular compartment-targeting pathways. However, it was found that the highest yield of patchoulol was achieved in transformant plants with similar transcription levels of FPS and PTS. Also, the optimized transcription ratio between PTS and FPS is determined at 1.12 based on statistical analysis and model simulation.

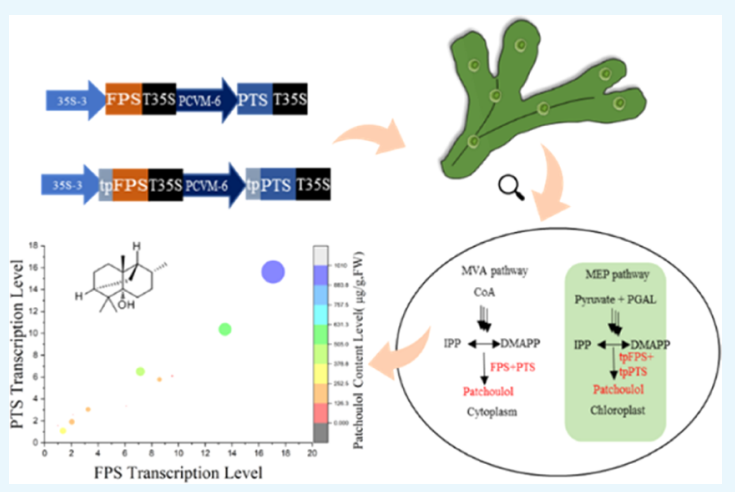
Therefore, two kinds of new optimized pathway vectors were constructed. One is based on the fusion protein method, and the other is based on protein expression individually, in which the same promoter and terminator were used to derive the expression of both FPS and PTS. The effect of pathway optimization was tested by transient and stable transformation. The production of patchoulol in transient transformation was the same for the two abovementioned kinds of matching pathway and higher than that for the original pathway. Also, in stable transformation, the yield of patchoulol reached up to $3250.30 \mu \mathrm{g} / \mathrm{g}$, being three times the maximum content before optimization. It is suggested that M. paleacea is a powerful plant chassis for terpenoid synthetic biology and the matching between enzymes may be the key factor in determining the metabolic flux of the pathway in the study of synthetic biology.

\section{INTRODUCTION}

Plant secondary metabolites, such as terpenoids, phenolics, polyketides, and alkaloids, are structurally diverse with potential biology activity and excellent chemical properties, while these products are usually present in low abundance. ${ }^{1}$ Many researchers have attempted to efficiently produce plant secondary metabolites in microbial chassis such as Escherichia coli and yeast. Martin et al. $^{2}$ established an E. coli strain to produce amorpha-4,11-diene, the precursor of malaria treatment drug artemisinin, in large amounts $(25 \mathrm{mg} / \mathrm{L})$ by introducing the MVA pathway and amorpha-4,11-diene synthase $(A D S)$ with other several modifications. This procedure was used in a commercial scale in 2013 by Paddon et al. $^{3}$

Patchoulol is a sesquiterpene alcohol present in the patchouli oil extracted from the patchouli (Pogostemon cablin) leaves. ${ }^{4}$ Patchoulol is widely used in the cosmetics industry because of its peculiar and long-lasting scent. Besides, patchoulol also has many pharmacological functions such as antibacterial, ${ }^{5}$ anti-inflammatory, ${ }^{6}$ and antiviral capabilities. PTS (patchoulol synthase), the key enzyme of the patchoulol synthesis pathway, has been characterized. 8 ,9 Because of the finite natural plant resources and the limited yield of the extraction procedure, biotechnological engineering on different chassis plays a more important role in the patchoulol heterologous production. ${ }^{10}$

Several articles have reported on patchoulol heterologous production using the recombinant microbial platform. Patchoulol $(11.5 \mathrm{mg} / \mathrm{L})$ was harvested by suppressing ERG9 expression in engineered Saccharomyces cerevisiae, harboring the PTS gene. ${ }^{11}$ Also, the yield of patchouli can be increased to about five times in $S$. cerevisiae by expressing the patchoulol synthase gene (PTS) and farnesyl pyrophosphate synthase (FPS) as a fusion protein. ${ }^{12}$ The global metabolic engineering strategy (GMES) also was used to engineer the Mevalonate pathway in S. cerevisiae, in which $0.05 \mathrm{mg}$ of patchoulol could be produced for every gram of glucose consumed. ${ }^{13}$ Furthermore, the combination metabolic engineering strategy,

Received: September 14, 2020

Accepted: November 20, 2020

Published: December 16, 2020 
overexpressing PTS and its precursor gene while suppressing the carotenoid-like byproduct pathway, could efficiently improve the carbon flux and produce patchoulol at $60 \mathrm{mg} /$ L. ${ }^{14}$ The highest yield, $466.8 \mathrm{mg} / \mathrm{L}$, of patchoulol in engineered yeast was reported by suppressing the squalene pathway and overexpressing PTS and transcription factor UPC2-1 genes. ${ }^{15}$

Plant chassis harbors great potential for synthetic biology, in that photosynthesis can provide sufficient energy and substances, field cultivation costs are low, and plant-specific storage organisms can provide storage places for metabolites. However, using the terpenoid synthetic biology approach in plant chassis is challenging because of our limited understanding of plant genetic networks and increased complexity due to multicellularity. The tobacco plant Nicotiana tabacum has been used for patchoulol heterologous production; 0.03 $\mathrm{mg} / \mathrm{g}$ yield was obtained in the leaves. Targeting the sesquiterpenoid synthase to the plastids is the initial improvement of tobacco chassis, which increases the yield by 4000 fold. ${ }^{16}$ HMGR is the key regulatory enzyme in the Mevalonate pathway, ${ }^{17}$ and increasing its activity to redirect more carbon flux to target terpenoid precursors is a potential strategy. For example, by overexpressing truncated HMGR in Physcomitrella patens and targeting the PTS and FPS enzymes to plastids, the yield of patchoulol was raised to $1.34 \mathrm{mg} / \mathrm{g}$ dry weight. ${ }^{18}$ It is the highest yield reported for patchoulol heterologous synthesis based on plants so far. Yet, the production is still limited.

Though plants are a potential chassis for terpenoid metabolism engineering, working with plants is beset with problems because of slow generation times, genetic redundancy, and difficulties in transformation, compared to microbes. To circumvent these difficulties, we adopted Marchantia as a test bed to study terpenoid synthetic biology in plants. This liverwort plant system is fast and easy to culture and transform. Marchantia paleacea https://baike.baidu.com/ pic/\%E 5\% 9 C\%B0\%E9\% 92\%B 1/4906381/1321988/ 0 e 2442 a 7 d 933 c8954510fb 8 bd 11373 f0830200d 7 fr $=$ lemma\&ct=cover can reproduce both sexually and asexually, and regenerated plants spontaneously produce clonal propagules within a few weeks of an experiment. ${ }^{19}$ In addition to sexual reproduction, $M$. paleaceahttps://baike.baidu.com/pic/ \% E 5 \% 9 C \% B 0 \% E 9\% $92 \%$ B 1 / 4906381 / 1321988 / $0 \mathrm{e} 2442 \mathrm{a} 7 \mathrm{~d} 933 \mathrm{c} 8954510 \mathrm{fb} 8 \mathrm{bd} 11373 \mathrm{f0} 830200 \mathrm{~d} 7 \mathrm{fr}=$ lemma\&ct=cover can be vegetatively propagated by germs; each germ originates from a single cell and can develop into a complete plant. $^{20}$ Through germ reproduction, many plants with the same genetic background as the parent can be quickly obtained, effectively reducing the interference of genetic factors. The plant is a haploid and has a genome of about $220 \mathrm{Mb}$, which shows low genetic redundancy in the regulation of most pathways. ${ }^{21}$ Meanwhile, a simplified Agrobacteriummediated transformation method for sporelings of Marchantia polymorpha (Agar-Trap) has been explored. It is reported to have a high transformation ratio of up to $100 \% .{ }^{20}$ Moreover, M. paleacea contains oil bodies rich in sesquiterpene, diterpenoid, and aromatic compounds, ${ }^{22}$ which suggested that $M$. paleacea could provide a potential store site for terpenoids in plant chassis.

Here, we reported the construction and optimization of patchoulol pathways in M. paleacea to explore the heterologous production of patchoulol in plants and studied the factors determining the metabolic flux of the patchoulol pathway, such as cellular compartment targeting, the expression level of each enzyme, and the matching between enzymes in the pathway in M. paleacea.

\section{RESULTS}

Transformants in M. paleacea. We modified the $35 \mathrm{~s}$ and CVM promoter and selected $35 \mathrm{~s}-3$ and cvm- 6 as the strong promoters in this experiment through the Dual-Luciferase Reporter Assay System (related data were not shown). GV3101, harboring the plasmids PDGB3::p35s-3-PTST35s:CVM-6-FPS-T35s:35S-HYG-T35s and PDGB3::p35s-3tpPTS-T35s:CVM-6-tpFPS-T35s:35S-HYG-T35s, was used to introduce PTS and FPS into the germ via Agrobacteriummediated transformation method. After Agrobacterium transformation and Hygromycin selection, the survival $T_{0}$ generation germs were placed in the selection medium. Then, we placed $T_{1}$ generation and wild-type gemmae in the selection medium again, and about 1 week later, the wild-type germs turned white and lost their vitality (Figure 1). Among

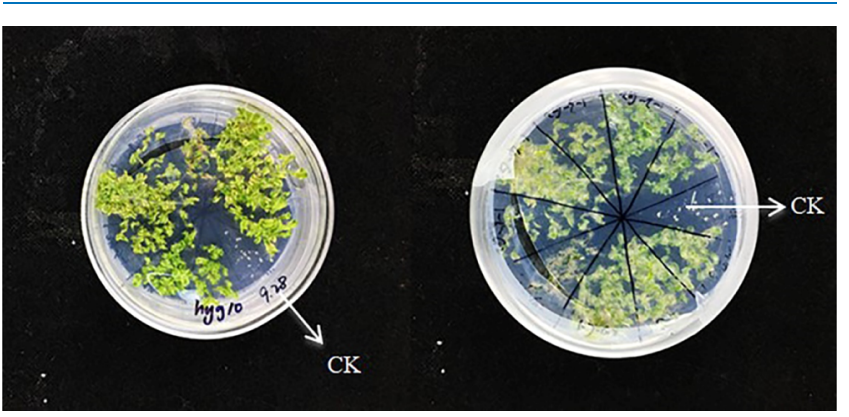

Figure 1. Resistant seedlings and wild-type seedlings screened in the medium.

them, 17 independent transgenic plants (10 in the tpFPS + tpPTS group and 7 in the FPS + PTS group) were obtained and confirmed to have PTS and FPS using the PCR test. They were named as $1-1,1-2,1-3,1-7,1-9,1-10,1-12,1-13$, $1-14,1-15,2-1,2-2,2-3,2-5,2-8,2-10$, and $2-11$, respectively. It demonstrated that FPS and PTS have been successfully integrated into the $M$. paleacea genome, and the Agrobacterium-mediated M. paleacea https://baike.baidu.com/ pic/\%E5\% 9 C\%B 0\%E9\%92\%B 1/4906381/1321988/ $0 \mathrm{e} 2442 \mathrm{a} 7 \mathrm{~d} 933 \mathrm{c} 8954510 \mathrm{fb} 8 \mathrm{bd} 11373 \mathrm{f} 0830200 \mathrm{~d} 7 \mathrm{fr}=$ lemma\&ct=covergerm transformation method is feasible (Figure 2).

$\mathrm{T}_{0}$ generation transformants' gemmae were selected using subculture medium containing Hygromycin. About 1 week later, the CK (wild-type plant) and the false-positive transformants will die and the transformants could be obtained.

Production of Patchoulol in $M$. paleacea Was Feasible. To test whether the transgenic group could produce patchoulol, gas chromatography-mass spectrometry (GCMS) analysis was performed. As the chromatogram shows (Figure 3), the same peak appeared in the standard sample and transgenic group at about $7.46 \mathrm{~min}$, except the wild-type sample. Then, the mass spectrum was extracted at the corresponding chromatographic peak, and the feature fragments were identified. Thus, the target product could be synthesized by exogenous gene insertion, transcription, and translation in $M$. paleacea https://baike.baidu.com/pic/ \% E 5 \% 9 C \% B 0 \% E $9 \% 92 \%$ B 1/ $4906381 / 1321988$ / 


\section{Cytoplasm}

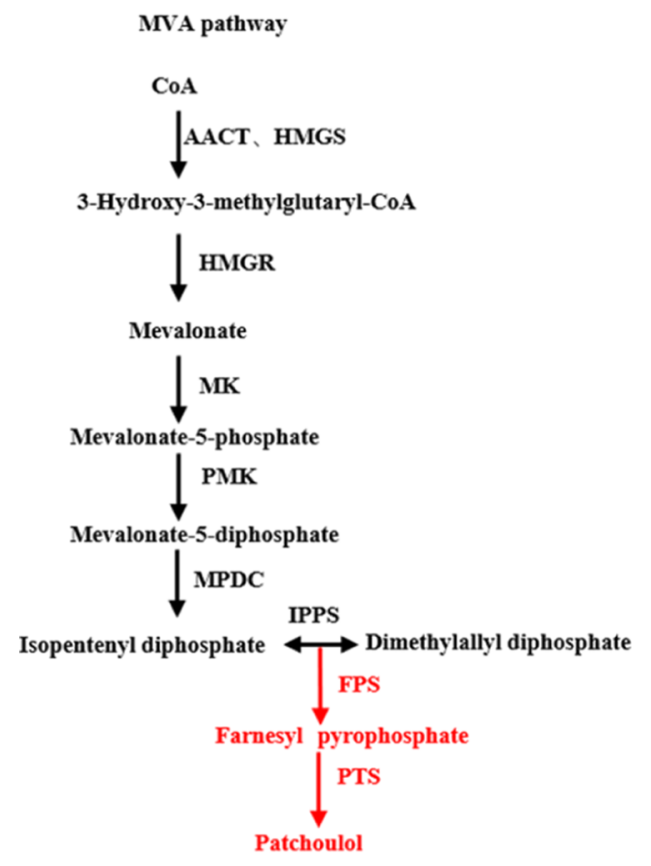

\section{Chloroplast}

MEP DXS pathway

Pyruvate + D-Glyceraldehyde 3-phosphate

$\downarrow$ DXS、DXR

2-C-Methyl-D-erythritol 4-phosphate

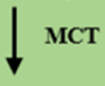

4-(Cytidine 5'-diphospho)-2-C-methyl-D-erythritol

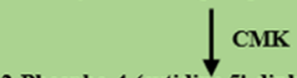

2-Phospho-4-(cytidine 5'-diphospho)-2-C-methyl-D-erythritol

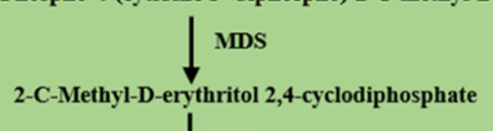

HDS

4-Hydroxy-3-methylbut-2-enyl-diphosphate

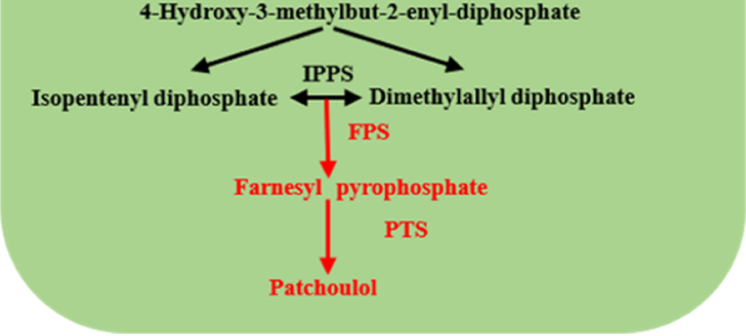

Figure 2. Patchoulol synthesis pathway in Marchantia paleacea.

a

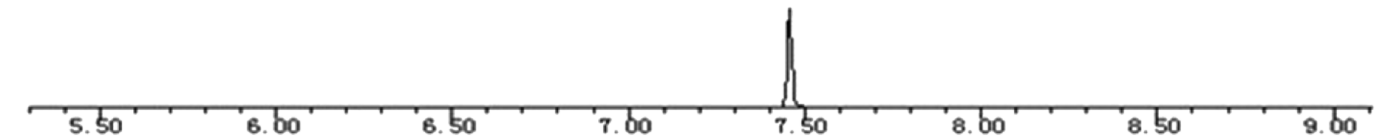

b

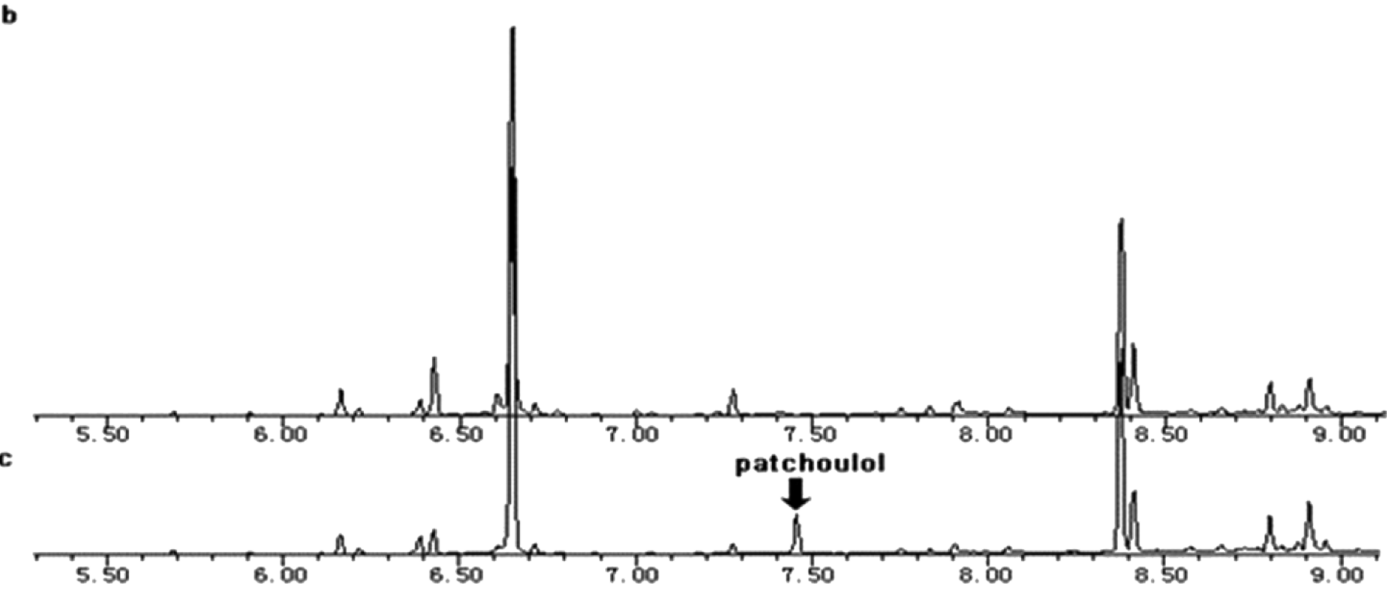

Figure 3. GC-MS chromatograms of (a) patchoulol standard; (b) wild-type Marchantia paleacea; and (c) transgenic Marchantia paleacea. Also, this figure is the total ion current diagram (TIC), the $x$ axis is the retention time, and the $y$ axis is the signal response value.

$0 \mathrm{e} 2442 \mathrm{a} 7 \mathrm{~d} 933 \mathrm{c} 8954510 \mathrm{fb} 8 \mathrm{bd} 11373 \mathrm{f0} 830200 \mathrm{~d} 7$ ? fr $=$ lemma\&ct=coverL. Compartmentalized metabolic engineering has proven to be an effective strategy for improving sesquiterpene production. The plant material was divided into two groups: FPS + PTS group and tpFPS + tpPTS group; the $t p F P S+t p P T S$ group showed a higher patchoulol content on average according to GC-MS quantitative analysis. Also, $1-9$ in the tpFPS + tpPTS group and $2-3$ in the FPS + PTS group yielded the maximum production of 1006.45 and 621.56 $\mu \mathrm{g} / \mathrm{g}$ dry weight of patchoulol, respectively. Though the tpFPS + tpPTS group showed higher average yield, there was no significant statistical difference between the two groups via student's test, defining the significant level as $P<0.05$. It is suggested that the chloroplast could provide a suitable microenvironment for FPP accumulation by the MEP pathway, which could improve patchoulol synthesis and accumulation. 


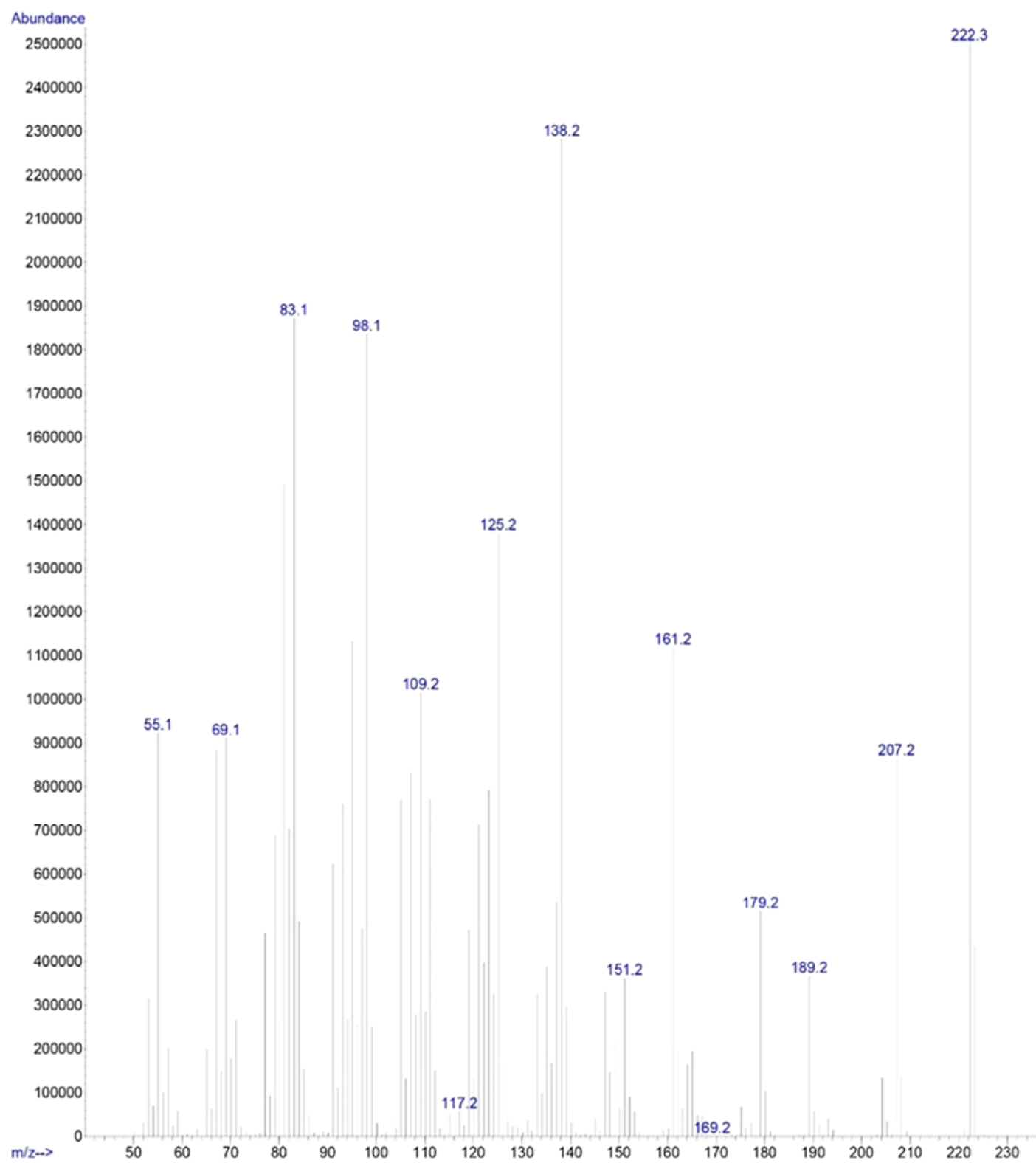

Figure 4. Mass spectrum of transgenic plants. $m / z=98,138,161$, and 222 were selected as the diagnostic ions to quantify patchoulol.

Also, the production of patchoulol in M. paleacea was feasible (Figure 4).

The pathway marked in red was the patchoulol heterogeneous pathway introduced into M. paleacea via Agrobacterium transformation. Two terpenoid pathways could provide the common precursor IPP (Isopentenyl diphosphate) in different cell compartments: cytoplasm and chloroplast; it was feasible to produce patchoulol in M. paleacea. The compartmentalization strategy was performed using signal peptides; the cytoplasm lines and chloroplast lines were named as FPS + PTS and tpFPS + tpPTS groups, respectively (Figure 5).

Cell Compartmentation Has No Significant Effect on the Transcription Level. In the tpFPS + tpPTS and FPS + PTS groups, FPS appears to show a higher transcription level than PTS; it demonstrated that FPS is the committed enzyme in the plastid patchoulol pathway and regulated the PTS transcript level. Compared to others, 1-9 was the highest in these lines (Figure 6). As for the FPS + PTS group, FPS was higher than PTS in most samples, and 2-3 was the highest

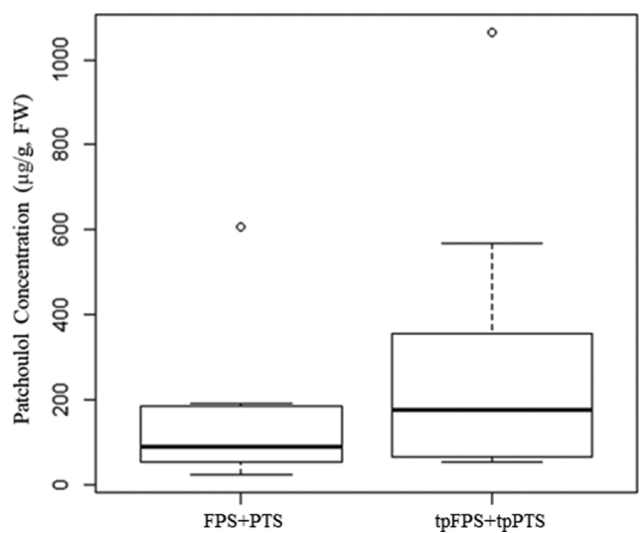

Figure 5. Content of patchoulol in the FPS + PTS group and tpFPS + tpPTS group The statistical analysis significance was calculated using the student's test, defining the significant level as $P<0.05$. 

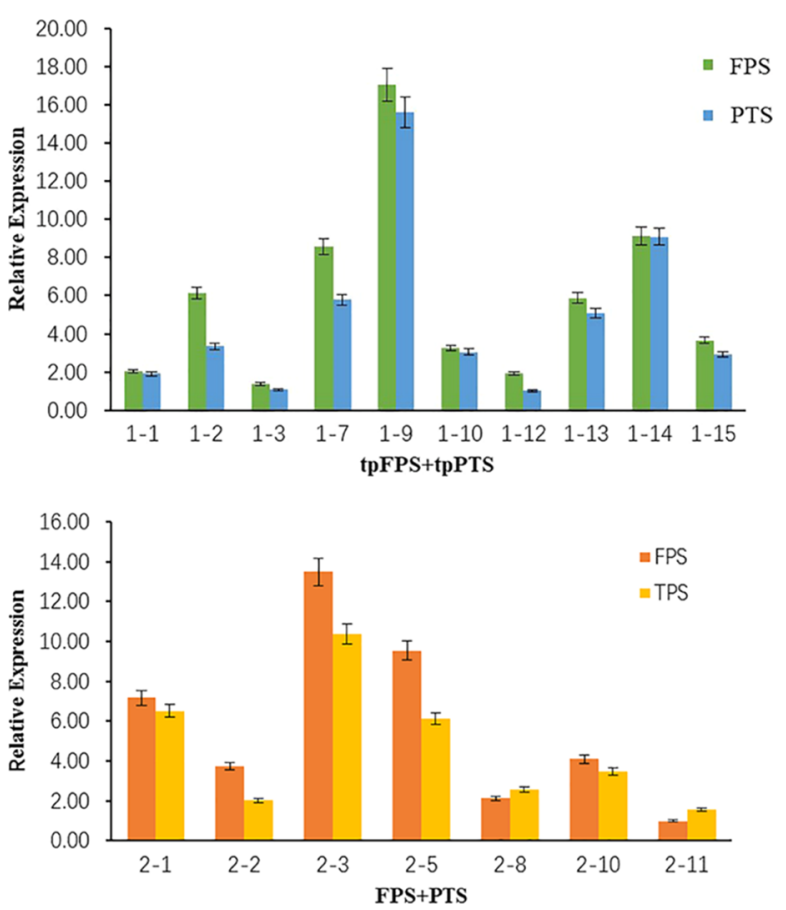

Figure 6. Relative expression of patchoulol pathway genes (FPS and $P T S)$ in the tpFPS + tpPTS and FPS + PTS groups. Error bars are shown as SE $(n=3)$.

individual. In this study, Arabidopsis RuBisCO, a small subunit transit peptide, carried out the pathway plastid targeting and compartmentation strategy. However, the insertion position is the vital factor affecting the transcription level except the transcript units themselves. When comparing the FPS and PTS transcription levels in the tpFPS + tpPTS and FPS + PTS groups, it seemed that there was no significant difference between different groups, whether FPS or PTS. Overall, it was suggested that the compartmentation strategy was not the key factor affecting the transcription level in our study (Figure 7).

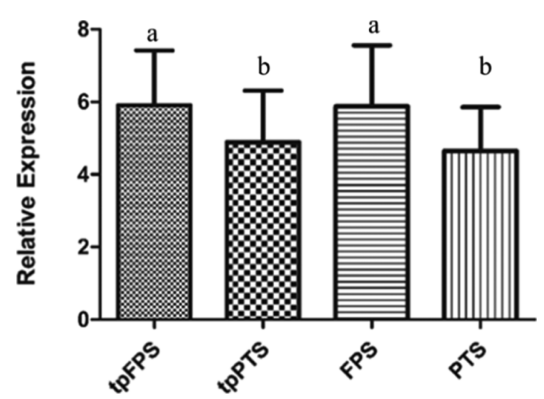

Figure 7. Different lines showing FPS and PTS average relative expression levels. Bar shows the average of different groups, FPS, PTS, and their transcription level + SE. The statistical analysis significance was calculated using the student's test, defining the significant level as $P<0.05$.

Dynamic Changes in the Yield and Transcription Level. To explore the dynamic changes during the plant growth period, we analyzed the relative expression and metabolism in 2-3 and 1-9 lines (Figure 8). With $M$. paleacea growth, FPS and PTS expression levels increased continually and FPS was higher than PTS in same lines as well as in different lines. The addition of plasmid signal peptide tp enhanced gene expression to a certain extent from 1 to 4

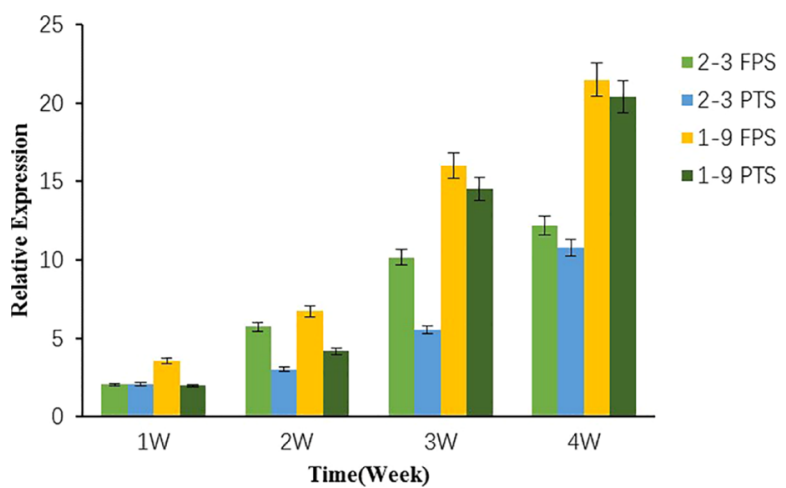

Figure 8. Dynamic analysis of $1-9$ and 2-3 mRNA relative expression levels.

weeks. Because of aging, the RNA extraction seemed difficult for transcription level analysis after 4 weeks. However, the constitutive promoters $35 \mathrm{~s}$ and CVM promoted the genesustained expression throughout the plant growth cycle. The patchoulol content increased for 5 weeks, 2-3 content declined at the 6th week, and 1-9 content declined gradually at 7 th week and then declined rapidly. The highest accumulation of content happened at the 5th week in the $F P S+P T S$ group and at the 6th week in the tpFPS + tpPTS group; the yields were 621.56 and $1006.45 \mu \mathrm{g} / \mathrm{g}$, respectively. According to the abovementioned data, a regular pattern showed that the accumulated content of patchoulol synthesis transgenic liverworts reached its peak at 5-6 weeks and then declined (Figure 9).

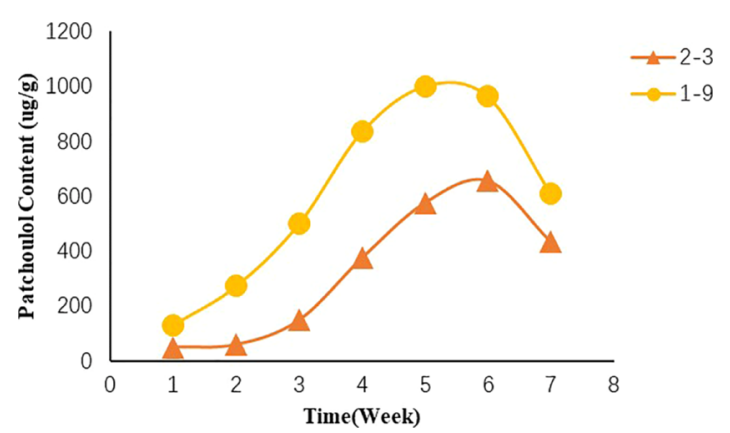

Figure 9. Dynamic analysis of 1-9 and 2-3 patchoulol content.

Pathway Matching: the Transcription Ratio Determined the Efficiency. Although the patchoulol synthesis pathway is short and simple, it is a good way to explore the efficiency of pathway matching. First of all, every module in the pathway should be reasonably designed and matched. In detail, strict control of transcript unit's (TU's) transcription level in the modules might be accomplished via optimal DNA parts such as promoters, terminators, CDS, and even transcription factor. In this study, we guessed that the patchoulol content was related to the gene transcription level, which was reasonably matched in the pathway; however, the key point was not clear. Then, we established a scatter plot of yields and different gene expressions to describe the connection between them (Figure 10). It seemed that the transcript level ratio FPS/ PTS plays a vital role. Many spots, as shown in the figure, were around 1, which demonstrated that the similar transcription level between FPS and PTS was the rational match and could contribute to the final product. We hypothesize that when the 


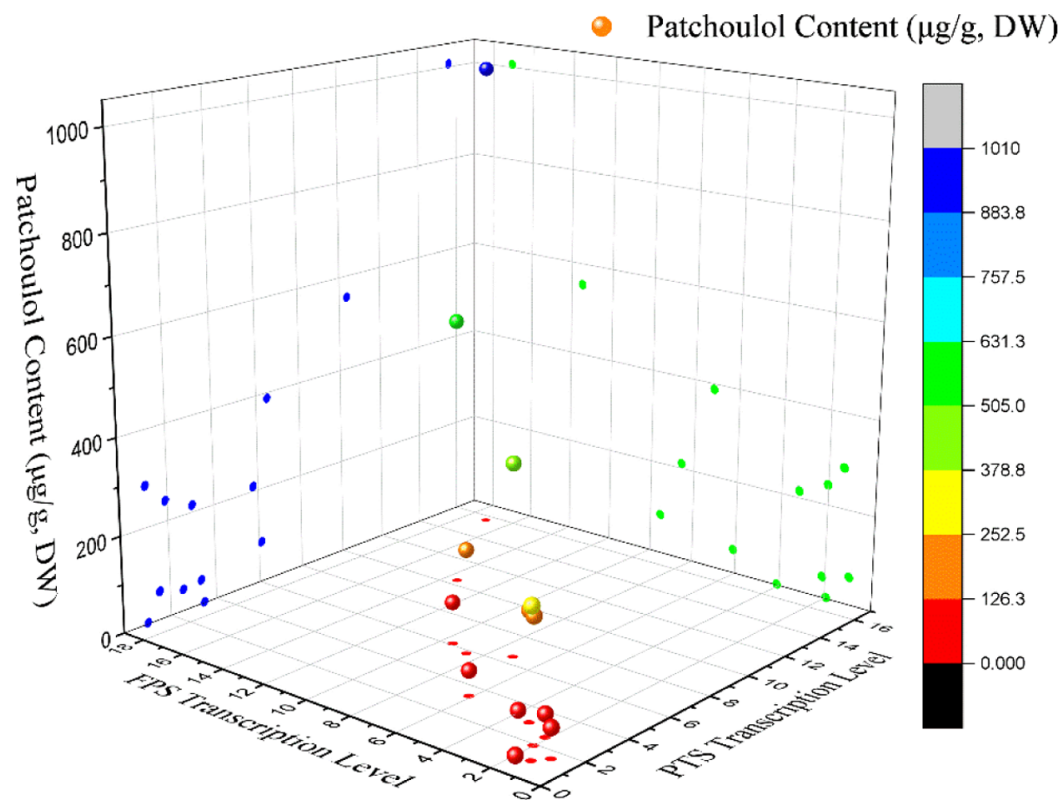

Figure 10. Relationship between the patchoulol content and transcript level. The relationship is described by the three-dimensional plot; the ball represents the patchoulol content. The color map on the right is used to show the patchoulol content level, and the ball color is related to the color map; DW: dry weight.

ratio was about 1 , the optimal product could be obtained. Then, a model was established to prove the hypothesis in this study.

According to the steady-state hypothesis of metabolites in metabolic flux analysis, we can assume that each metabolic reaction is in a steady state, that is, the consumption rate and production rate of each metabolite are equal. ${ }^{23}$ The production rate of the final product in the metabolic network is related to the rate-limiting step in the pathway. The rate-limiting step is the reaction with the lowest enzyme catalytic activity. The catalytic ability can be expressed by the reaction velocity $v$. The constant $k$ was used to describe the relationship between the reaction velocity $v$ and relevant gene transcription level.

$v_{F P S}$ : FPS reaction velocity; $v_{P T S}$ : PTS reaction velocity; $v$ : patchoulol production velocity P: patchoulol content; TR: transcript level; av: actual value; and tv: theoretical value.

In the patchoulol pathway, the production velocity of the final product was determined by lower reaction velocity between FPS and PTS.

$$
\begin{aligned}
& v=\left\{\begin{array}{l}
v_{F P S}, v_{F P S}<v_{P T S} \\
v_{P T S}, v_{F P S} \geq v_{P T S}
\end{array}\right. \\
& v=\min \left[v_{F P S}, v_{P T S}\right]
\end{aligned}
$$

then we defined $v$ as $v=k \cdot T R$

according to eqs 2 and $3, v=\min \left(k_{1} T R_{F P S}, k_{2} \cdot T R_{P T S}\right) P=\int v \mathrm{~d} t$ $=\int \min \left[k_{1} T R_{F P S}, k_{2} \cdot T R_{P T S}\right] \mathrm{d} t \approx \overline{\min }\left[k_{1} T R_{F P S}, k_{2} \cdot T R_{P T S}\right]$.

Then we calculated the $\min \left(P_{\mathrm{av}}-P_{\mathrm{tv}}\right)^{2}$ by solver function in the office and got the minimum value, which means the highest patchoulol content could be obtained when the transcript level ratio was about 1.12. This is consistent with our previous assumption.

Two Methods to Test the Pathway Matching Hypothesis in Patchoulol Synthesis. To test the applicability of the pathway matching conclusion derived from the hypothesis and the model, two kinds of vectors were constructed and introduced into $M$. paleacea via Agrobacterium-mediated stable and transient transformation.

The 35S-3 promoter was used to construct the FPS and PTS fusion protein vector and the same promoter-promoter vectors. Because of the time and transformation difficulty, relevant data were collected on a transient transformation group: p35s-3-FPS-linker-PTS-T35s, p35s-3-FPS-T35s:p35s-3PTS-T35s; and stable transformation group: p35s-3-tp-FPST35s:p35s-3-tp-PTS-T35s. We found that in the transient transformation group, the fusion protein line and p35s-3-FPST35s:p35s-3-PTS-T35s line could produce the same level of patchoulol content, about $15.34 \mu \mathrm{g} / \mathrm{g}$ (dry weight) (Figure $11)$, and higher than the original pathway $(5.03 \mu \mathrm{g} / \mathrm{g})$. What is more exciting is that the stable transformation group produced a maximum content of about $3250.30 \mu \mathrm{g} / \mathrm{g}$ (dry weight) patchoulol (Figure 12). It demonstrated that the fusion protein and the same promoter-promoter pathway might have the same effect, and the stable transformation of these two lines

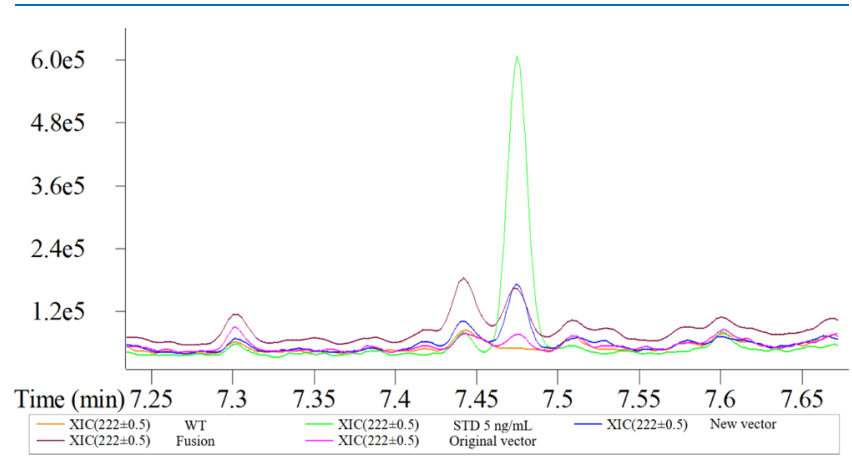

Figure 11. Transient transformation group GC-MS chromatogram. WT: wild type; STD $5 \mathrm{ng} / \mathrm{mL}: 5 \mathrm{ng} / \mathrm{mL}$ patchoulol standard sample; fusion: p35s-3-FPS-linker-PTS-T35s; new vector: p35s-3-FPST35s:p35s-3-PTS-T35s; and original vector: p35s-3-FPST35s:PCVM-6-PTS-T35s. The scFv linker (GenBank: BAA08905.1) was used to fuse the FPS and PTS protein. 


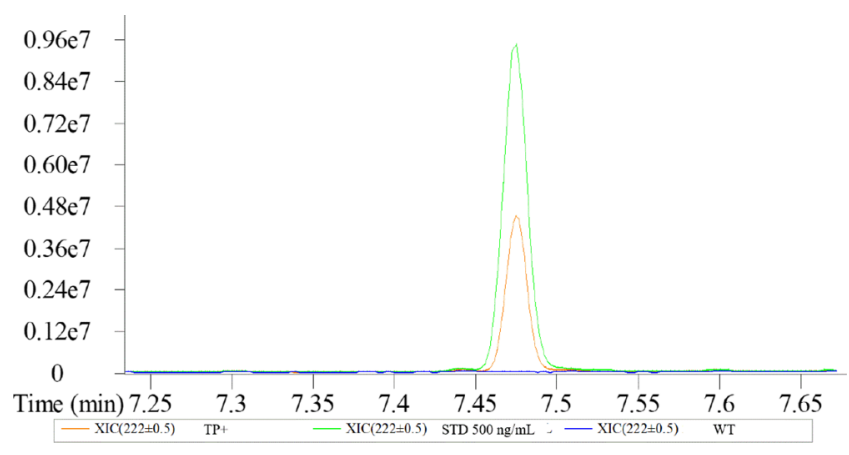

Figure 12. GC-MS chromatogram showing the stable transformation group. TP+: p35s-3-tp-FPS-T35s:p35s-3-tp-PTS-T35s; WT: wild type; and STD $500 \mathrm{ng} / \mathrm{mL}: 500 \mathrm{ng} / \mathrm{mL}$ patchoulol standard sample.

might provide higher yields than p35s-3-tp-FPS-T35s:p35s-3tp-PTS-T35s lines. Thus, the pathway matching hypothesis could be verified by fusion protein and the same promoterpromoter pathway.

\section{DISCUSSION}

Synthetic biology of important terpenoids generally focuses on the microorganism chassis, and compared with microbial synthetic biology, plant synthetic biology has great prospects but also has bottlenecks. M. paleacea, which is of small genetic size $^{21}$ and which grows fast, is a platform in our study because of the low genetic redundancy and mature and convenient genetic transformation method. Many attractive synthetic technological tools have been developed for studying $M$. paleacea, including those that improve the robust Loop assembly vector systems for nuclear and chloroplast transformation and genome editing. ${ }^{24}$ Here, we engineered the patchoulol pathway via compartmentalizing FPS and PTS in the chloroplasts of $M$. paleacea. Combining the compartmentalization strategy with enhancement of precursors, we successfully obtained 1006.45 and $621.56 \mu \mathrm{g} / \mathrm{g}$ dry weight of patchoulol in tpFPS + tpPTS and FPS + PTS groups of $M$. paleacea thallus. In both tpFPS + tpPTS and FPS + PTS groups, although the same expression vectors and retargeting and overexpression strategies were used, the examination on the expression level of all lines revealed the low expression level, which explains the low yield. The positional effects on the $M$. paleacea genome are much less understood. On the whole, the production of sesquiterpene patchoulol in $M$. paleacea was feasible and had potential.

In our study, we found that the introduction of the exogenous pathway may match or interfere with the endogenous terpenoid regulatory network, and the matching could determine the synthesis efficiency. In addition, the compatibility of exogenous pathways and plant endogenous pathways is an ideal state for efficient synthesis. A reasonable pathway consists of different optimal modules, and the biological element selection and match are the base of suitable modules. The biological elements include CDS, promoters, terminators, transcript factors, and so on and affect the pathway transcription level and even the translation level. In this study, we found that when the same transcription level was in the patchoulol pathway, the optimal yield was obtained in the tpFPS + tpPTS and FPS + PTS groups, which was similar to that of patchoulol in $S$. cerevisiae, ${ }^{12}$ and the yield could increase by five times via the fusion of FPS and PTS proteins because the fusion CDS could afford the same transcription level. It might be that the same transcription level between FPS and PTS was the reasonable match. The reasonable match means that the pathway could lead to consumption of FPP produced by overexpression in time to avoid triggering the chassis' own FPP regulatory network because FPP was strictly regulated in the cell. We guessed that the optimal yield will be obtained when the FPS and PTS transcription levels are same, then the hypothesis was verified via mathematical and consisted with the guess. After that, the fusion protein and same promoterpromoter vectors were constructed for transient or stable transformation. It is found that fusion protein afforded the same patchoulol yields with the same promoter pathway in the cytoplasm, higher than those with the original unoptimized pathway in the cytoplasm. Compared with the different promoter pathway, the same promoter pathway in plastid could increase the patchoulol yields by 3 times to about $3250.30 \mu \mathrm{g} / \mathrm{g}(\mathrm{DW})$. Thus, according to the transient and stable transformation data, we can infer that pathway matching could significantly improve the patchoulol production in $M$. paleacea.

Usually, overexpression of FPS to accumulate FPP in the cell sometimes might not work as we expected. FPP, which has been demonstrated to have toxicity in the E. coli cell, could inhibit cell growth, and the introduction of FPP sensor regulators could balance the endogenous regulatory network and heterogeneous pathway. ${ }^{25}$ Protein farnesylation is a posttranslational modification and central to molecular cell biology. ${ }^{26}$ Farnesyl transferase inhibitors are potential cancer anticancer agents, ${ }^{27}$ which correct aberrant genome organization in Hutchinson-Gilford progeria syndrome fibroblasts. $^{28}$ As for plants, many mechanisms of farnesylation signaling are still unknown. And the mutant of plant protein farnesyl transferase caused meristem or ganization and mediate brassinosteroid biosynthesis to regulate abscisic acid responses. $^{29}$ Thus, FPP was strictly regulated because it plays a critical role in the growth and development of organisms. Meanwhile, the codon-optimized genes have previously been shown to be useful for increasing the translation level. However, whether it is useful for the transcription level or not is not clear. ${ }^{30}$

The isoprene is production via MVA and MEP pathway in plant. Generally, the crossflow between the MVA and MEP pathway is restricted; nevertheless, the systematic analysis of isoprenoids in 86 plant species shows that strict compartmentalization of biosynthesis occurred in triterpenoids, tetraterpenoids, and diterpenoids. Contrary to that, monoterpenes, diterpenes, hemiterpenes, sesquiterpenes, and polyterpenes could be derived by both pathways in the case of specific environmental conditions. ${ }^{31}$ Compartmentalized metabolic engineering in Nicotiana $\operatorname{spp}^{16}$ and P. patens, ${ }^{18}$ especially targeting the isoprenoid synthase, has proven to be an effective strategy for improving sesquiterpene production such as patchoulol and artemisinin in plants. Also, the possible reason for retargeting terpenoids to FPP as the substrate in plastids that can effectively improve the yield may be the fact that the sesquiterpene production mainly relies on the MVA pathway operation on plastids ${ }^{32}$ and retargeting it to plastids could provide a less FPP-competitive environment. In this study, we co-expressed FPS and PTS in the cytoplasm and chloroplast. The chloroplast targeting of FPS and PTS lines affords a high maximum yield compared to the cytoplasm lines, which is consistent with the patchoulol production in $P$. patens. ${ }^{18}$ Although the plastid-targeting lines showed a high average 

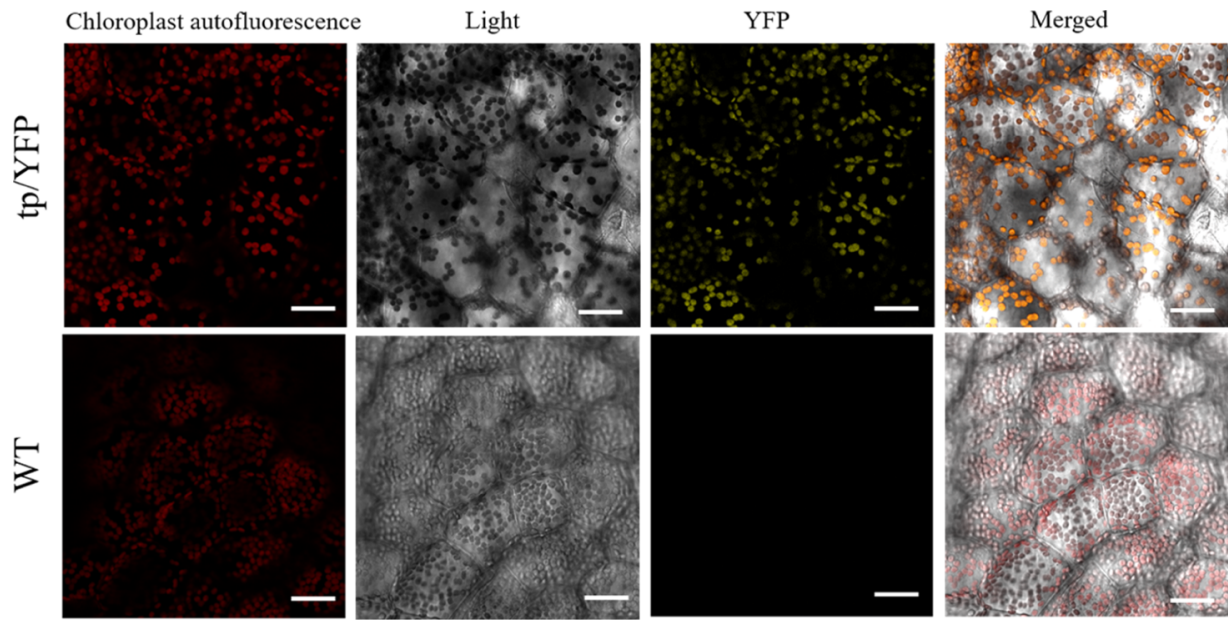

Figure 13. Intracellular organelle location. Fluorescence labeling of the tp/YFP protein.

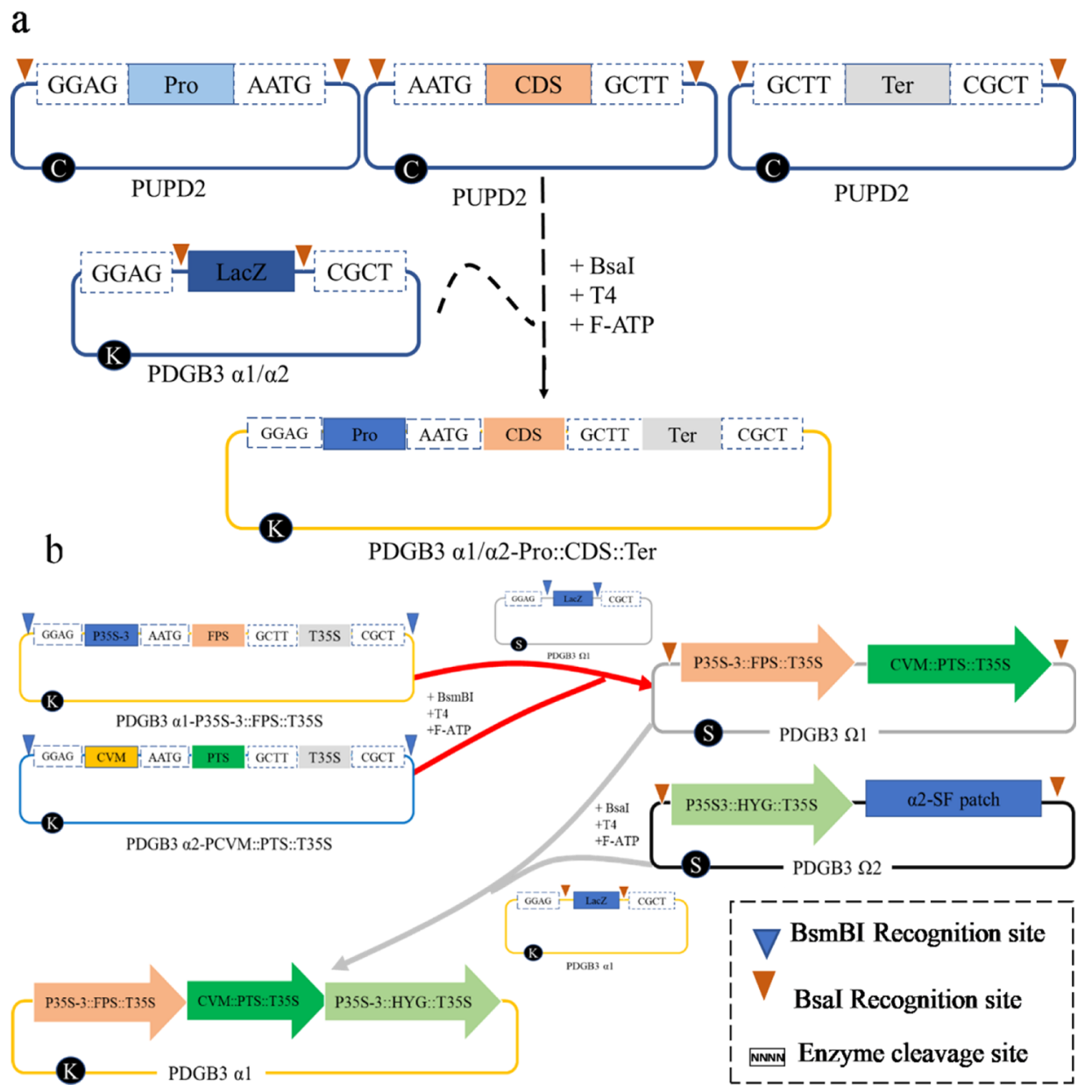

Figure 14. Binary assembly in the $\alpha$ or $\Omega$ level for patchoulol production. (a) Multipartite assembly of DNA parts promoter, CDS, and terminator for the construction of TU in an $\alpha$-level destination vector. Also, the tpFPS +tpPTS group vector was similar to this assembly process. (b) Binary assembly of the patchoulol pathway. Two kinds of TU, $\alpha$-level TU and $\Omega$-level TU, could be assembled into an $\Omega$ or $\alpha$ level vector, respectively. The $\alpha 2$-SF path has no specific functional DNA parts, loaded on the PDGB3 $\alpha 2$ empty vector for the change between $\alpha$ and $\Omega$ level TU. Also, the tpFPS $+t_{p P T S}$ group vector construction is the same as given above.

yield, there was no significant difference between two groups according to the $t$-test. Meanwhile, we noticed a phenomenon that the cell compartmentation strategy has no significant effect on the transcription level because the signal peptide works at the translation level and not at the transcription level.
Overall, the reasonable collocation of modules inside the pathway, coordination or matching, and compatibility between the pathway and the chassis itself are the initial factors we should focus on, and these strategies might help us to better understand plant chassis and design plant synthetic biology. 


\section{EXPERIMENTAL SECTION}

Materials and Methods. Plant Materials and Growth Conditions. A female strain of $M$. paleacea, FSN-2, was isolated from Hubei province (china) and maintained on 1/2 Gamborg media ( $0.5 \times$ strength Gamborg B5 medium plus vitamins, Duchefa Biochemie G0210, $\mathrm{pH} 5.8$ ) and $1 \%(\mathrm{w} / \mathrm{v})$ agar under sterile conditions, at $22^{\circ} \mathrm{C}$, with $10,000 \mathrm{l} \times$ light $(16 \mathrm{~h} \mathrm{light} / 8 \mathrm{~h}$ dark), in a $100 \mathrm{~mL}$ Erlenmeyer flask.

Vector Construction. GoldenBraid Kit 2, a kind of synthetic tool, based on the type IIS restriction enzymes, was used to assemble DNA elements. ${ }^{33}$ Three kinds of plant DNA part used in this paper are promoter with $5^{\prime}$ UTR (PROM5), coding sequence with start and stop codons (CDS), and $3^{\prime}$ UTR with terminator (3TERM). The $5^{\prime}$ end and $3^{\prime}$ fusion sites on each part were designed, following the common genetic syntax of phytobricks, which enables directional assembly of these three parts into the transcription unit (TU) in one reaction. All DNA parts were cloned into a universal acceptor plasmid called pUPD2, which has one pair of divergent BsmbI sites to clone a DNA part and one pair of convergent BsaI sites to assemble parts into a transcription unit.

Two strong PROM5 parts, PCVM-6 and P35S-3, were built based on the genome sequence of the Cassava vein mosaic virus and cauliflower mosaic virus by the synthetic strategy (protocol in the Supporting Information). The expression level of these new promoters was measured by the Dual-Luciferase Reporter Assay System (Promega), following the recommended conditions (Figure S1).

CDS parts encoding the FPP synthase (FPS) and patchoulol synthase (PTS) were domesticated based on the genome of Gallus gallus and P. cablin by the synthetic strategy (protocol in the appendix). The sequence encoding Arabidopsis $\mathrm{RuBisCO}$, a small subunit transit peptide, was fused to the $5^{\prime}$ terminal of these CDS to build two new plastid-targeting CDS (Figure $13)$, called as tp + FPS and tp + PTS. CDS of the hygromycin phosphotransferase gene ( $H Y G$ ) provided by the GoldenBraid kit were used as a selectable marker for Marchantia.

T35S, provided by the GoldenBraid kit, was used as a terminator part in this paper. In a single step reaction with BsaI and T4 ligase, three parts, PROMOTER5, CDS, and 3TERM, can be assembled into a transcription unit and cloned into $\alpha$ level destination vector PDGB3. Two TUs, P35S-3-FPS-T35S and PCVM-6-PTS-T35S, harboring in $\alpha 1$ and $\alpha 2$ PDGB3, respectively, can be assembled together into $\Omega 1$ PDGB3. In the same way, the hygromycin resistant TU, P35S-3-HYGT35S, was assembled with Twister plasmid pDGB1alpha2_SF (GB0107) and cloned into $\Omega 2$ PDGB3. Then, DNA assemblies harbored in these two $\Omega P D G B 3$ can be joined together and cloned into $\alpha 1$ PDGB3 in a single step reaction with BambI and T4 ligase. The binary assembly can be done recursively, and the number of TU assembled doubles every loop (Figure 14).

Agrobacterium-containing plasmids were introduced into the leaf of M. paleacea by transient transformation. Scale bar indicates $30 \mu \mathrm{m}$; YFP: yellow fluorescent protein.

Transformation Procedures. The plasmid-harboring patchoulol pathway was introduced into Agrobacterium tumefaciens GV3101, by which the T-DNA between RB and LB was transformed into Marchantia via the Agar-Trap method. ${ }^{20}$

Gemmae produced in a 3-week thallus gemma cup were placed in precultured solid medium and incubated for 4 days under the normal growth conditions mentioned above. Precultured solid medium consisted of $0.5 \times$ Gamborg media, $1 \%$ sucrose, and $1 \%$ agar and was autoclaved. For each interest plasmid, a single Agrobacterium colony was incubated into 1 $\mathrm{mL} \mathrm{LB}$ medium without antibiotics and then inoculated into $15 \mathrm{~mL} \mathrm{LB}$ medium plus the antibiotics at $28^{\circ} \mathrm{C}$ with shaking at $200 \mathrm{rpm}$ for 1 day. The Agrobacterium cultures, induced for 3 h with $150 \mu \mathrm{M}$ acetosyringone, were centrifuged for $10 \mathrm{~min}$ at $4000 \mathrm{~g}$, resuspended in $10 \mathrm{~mL}$ transformation solution, and then incubated for $3 \mathrm{~h}$ at $28{ }^{\circ} \mathrm{C}$ at $200 \mathrm{rpm} ; \mathrm{OD}_{600}$ was adjusted between 0.5 and 0.8 . The transformation solution consisted of $10 \mathrm{mM} \mathrm{MgCl}$, $10 \mathrm{mM}$ MES-NaOH, $0.01 \%$ Tween 20, $150 \mu \mathrm{M}$ AS. Then, the resuspended Agrobacterium was mixed with precultured gemmae and kept still for $10 \mathrm{~min}$. The infected gemmae were placed on the $0.5 \times$ Gamborg B5 media and $150 \mu \mathrm{M}$ AS plates and, after 2 days dark coculture with $1 \mathrm{~mL}$ of screen solution and $100 \mu \mathrm{g} / \mathrm{mL}$ cefotaxime and $20 \mu \mathrm{g} / \mathrm{mL}$ hygromycin, were spread on the medium to screen the transformants. After about 2 weeks, the transformants $\left(T_{0}\right.$ generation) were transferred to the subculture $0.5 \times$ Gamborg B5 media and $100 \mu \mathrm{g} / \mathrm{mL}$ cefotaxime and $10 \mu \mathrm{g} / \mathrm{mL}$ hygromycin. $\mathrm{T}_{0}$ generation gemmae were then cultured with low humidity and strong light; after 1 month, the surviving grams were hypothetical homozygous transgenic plants $\left(T_{1}\right.$ generation).

Transient transformation was used to detect the promoter activity, the subcellular localization of the signal peptide, and the effect of the vector reconstructed according to the matching hypothesis. The thallus was cut into pieces and then infected with Agrobacterium according to the stable transformation process. After 2 days of cocultivation, the DualLuciferase data and patchoulol content data were collected. As for the subcellular localization, gemmae cocultured for 2 days were washed with sterile water and then observed using a confocal microscope.

Screening of Transformants with PCR. Two weeks old fresh liverwort samples were harvested, and the transgenic liverwort was identified by PCR analysis using genomic DNA isolated by the TPS method. The primers are shown in Table 1.

Table 1. Primer Sequence Used in PCR Analysis

\begin{tabular}{|c|c|c|}
\hline type & primer & sequence $\left(5^{\prime}-3^{\prime}\right)$ \\
\hline \multirow{4}{*}{ PCR } & FPSF & ATGCAGCCCCATCATCATCATA \\
\hline & FPSR & GGGTCCCCAAAGCAGTCCAGGTAA \\
\hline & TPF & ATGGCTTCСTCTATGCTCTC \\
\hline & PTSF & АСАТСАСТTССАТСGCAAGCAAGG \\
\hline \multirow{7}{*}{$\begin{array}{r}\mathrm{RT} Q \\
\text { PCR }\end{array}$} & PTSR & ACATCACTTCCATCGCAAGCAAGG \\
\hline & FPSQF & GAAGGATGCTGAGAGCCTGCGGTG \\
\hline & FPSQR & ATGAGGTCCAGCAATCTGCCCGAGC \\
\hline & PTSQF & GATTGGGTGTTCTCCCGACCTCCT \\
\hline & PTSQR & TCTTTGTAAATAACCTCAAGTGTTCGGA \\
\hline & ACTINQF & AGCAGCATGAAGATTAAGGTTG \\
\hline & ACTINQR & ССTTGGAGATCCACATCTG \\
\hline
\end{tabular}

Expression Profiling in M. paleacea. Two weeks old thallus (100 mg) was extracted by an RNAprep pure Plant Kit (TIANGEN BIOTECH, Beijing China) according to the protocol provided. The RNA quality and concentration were determined by a Nanodrop2000 Spectrophotometer (Thermo Fisher Scientific). Real-time quantitative PCR was performed 
using a SuperReal PreMix Plus(SYNR Green) kit (TIANGEN BIOTECH, Beijing, China) according to the protocol provided and run at $95{ }^{\circ} \mathrm{C}$ for $15 \mathrm{~min}, 40$ cycles at $95{ }^{\circ} \mathrm{C}$ for $20 \mathrm{~s}$ followed by $55{ }^{\circ} \mathrm{C}$ for $20 \mathrm{~s}$ and $72{ }^{\circ} \mathrm{C}$ for $20 \mathrm{~s}$ on a lightcycle 96 System (BioRad, Germany). qPCR was performed with three biological replicates for each sample and three technical replicates for each biological sample. Primers used are listed in (Table 1). M. paleacea ACTIN was used as the reference gene, and the transcript level was calculated as follows: $\Delta \mathrm{CT}=$ $\mathrm{CT}(\mathrm{GOI})$ - CT(ACTIN). $\Delta \Delta \mathrm{CT}$ was normalized using $\Delta \mathrm{CT}$, and the relative change in gene expression is calculated by the $2^{-\Delta \Delta \mathrm{CT}}$ method.

Quantification of Patchoulol Alcohol by GC-MS. Four weeks old fresh thallus was harvested, cut into small pieces, snap-frozen, and then ground into fine powder; $500 \mathrm{mg}$ of powder was extracted with $3 \mathrm{~mL}$ of ethyl acetate, followed by vertexing and sonication $(50 \mathrm{~W})$ for $20 \mathrm{~min}$. The extract was filtered, and the mixture were re-extracted twice more according to the same method; after that, these extracts were pooled and concentrated into $1 \mathrm{~mL}$. Meanwhile, $10 \mathrm{~g}$ of fresh thallus was filtered using a vacuum pump and dried at $75{ }^{\circ} \mathrm{C}$ for $48 \mathrm{~h}$, and dry weights were measured to calculated the sample water contents. The GC-MS analysis method was obtained from the work by Zhang. ${ }^{18}$ GC-MS analysis was performed on a GC-MS 7890/5975C (Agilent) equipped with an LTM column module (DB-1MS) $(10 \mathrm{~m} \times 0.18 \mathrm{~mm}$ i.d. $\times 0.18 \mu \mathrm{m})$. The samples $(1 \mu \mathrm{L})$ were injected with a split ratio of 25:1 into an LTM (DB-1MS) column using the following temperature program: $50{ }^{\circ} \mathrm{C}$ (held for $1 \mathrm{~min}$ ), 50$320{ }^{\circ} \mathrm{C}\left(30^{\circ} \mathrm{C} / \mathrm{min}\right.$, held for $\left.1 \mathrm{~min}\right)$, and the total time is 11 min ( 2 min solvent delay). The oven temperature was $200{ }^{\circ} \mathrm{C}$ (held for $11 \mathrm{~min}$ ). The injector temperature of GC was 250 ${ }^{\circ} \mathrm{C}$. The ion source temperature of the mass spectrometer was $230{ }^{\circ} \mathrm{C}$, and the transfer line temperature was set at $250{ }^{\circ} \mathrm{C}$. Helium was used as a carrier gas at a constant flow rate of 0.7 $\mathrm{mL} / \mathrm{min}$. Data were acquired by EI+ with Selected Ion Monitor (SIM) mode. Diagnostic ions: 98, 138, 161, 222 and RT (5.00-9.00) min were selected as the detail of the SIM method to quantify patchoulol. Statistical analysis significance was calculated using the student's test, defining the significant level as $P<0.05$.

\section{ASSOCIATED CONTENT}

\section{SI Supporting Information}

The Supporting Information is available free of charge at https://pubs.acs.org/doi/10.1021/acsomega.0c04391.

All the DNA elements' sequence and vector construction methods and the Dual-Luciferase data (PDF)

\section{AUTHOR INFORMATION}

\section{Corresponding Author}

Zhiqi Miao - School of Agriculture and Biology, Shanghai Jiao Tong University, Shanghai 200240, China; 이이.org/ 0000-0003-1168-3655; Email: zqmiao@sjtu.edu.cn

\footnotetext{
Authors

Yaojie Zhang - School of Agriculture and Biology, Shanghai Jiao Tong University, Shanghai 200240, China; (1) orcid.org/0000-0003-0768-7274

Lu Zhou - School of Agriculture and Biology, Shanghai Jiao Tong University, Shanghai 200240, China
}

Kexuan Tang - School of Agriculture and Biology, Shanghai Jiao Tong University, Shanghai 200240, China

Meihui Xu - School of Agriculture and Biology, Shanghai Jiao Tong University, Shanghai 200240, China

Complete contact information is available at: https://pubs.acs.org/10.1021/acsomega.0c04391

\section{Notes}

The authors declare no competing financial interest.

\section{ACKNOWLEDGMENTS}

This work was financially supported by the National Key R\&D Program of China (2018YFA0900600).

\section{REFERENCES}

(1) Huang, B.; Guo, J.; Yi, B.; Yu, X.; Sun, L.; Chen, W. Heterologous production of secondary metabolites as pharmaceuticals in Saccharomyces cerevisiae. Biotechnol. Lett. 2008, 30, 1121-1137.

(2) Martin, V. J. J.; Pitera, D. J.; Withers, S. T.; Newman, J. D.; Keasling, J. D. Engineering a mevalonate pathway in Escherichia coli for production of terpenoids. Nat. Biotechnol. 2003, 21, 796-802.

(3) Paddon, C. J.; Westfall, P. J.; Pitera, D. J.; et al. High-level semisynthetic production of the potent antimalarial artemisinin. Nature 2013, 496, 528-532.

(4) Croteau, R.; Munck, S. L.; Akoh, C. C.; Fisk, H. J.; Satterwhite, D. M. Biosynthesis of the sesquiterpene patchoulol from farnesyl pyrophosphate in leaf extracts of Pogostemon cablin (patchouli): mechanistic considerations. Arch. Biochem. Biophys. 1987, 256, 56-68.

(5) Aleu, J.; Hanson, J. R.; Galán, R. H.; Collado, I. G. Biotransformation of the fungistatic sesquiterpenoid patchoulol by botrytis cinerea. J. Nat. Prod. 1999, 62, 437-440.

(6) Chen, M.; Zhang, J.; Lai, Y.; Wang, S.; Li, P.; Xiao, J.; Fu, C.; Hu, H.; Wang, Y. Analysis of Pogostemon cablin from pharmaceutical research to market performances. Expet Opin. Invest. Drugs 2013, 22, 245-257.

(7) Jing-Jin, H.; Hai-Ming, C.; Chu-Wen, L.; et al. Experimental study on antinociceptive and anti-allergy effects of patchouli oil. J. Essent. Oil Res. 2013, 25, 488-496.

(8) Deguerry, F.; Pastore, L.; Wu, S.; Clark, A.; Chappell, J.; Schalk, M. The diverse sesquiterpene profile of patchouli, Pogostemon cablin, is correlated with a limited number of sesquiterpene synthases. Arch. Biochem. Biophys. 2006, 454, 123-136.

(9) Munck, S. L.; Croteau, R. Purification and characterization of the sesquiterpene cyclase patchoulol synthase from Pogostemon cablin. Arch. Biochem. Biophys. 1990, 282, 58-64.

(10) Jones, C. G.; Moniodis, J.; Zulak, K. G.; et al. Sandalwood fragrance biosynthesis involves sesquiterpene synthases of both the terpene synthase (TPS)-a and TPS-b subfamilies, including santalene synthases. J. Biol. Chem. 2011, 286, 17445-17454.

(11) Asadollahi, M. A.; Maury, J.; Møller, K.; Nielsen, K. F.; et al. Production of plant sesquiterpenes inSaccharomyces cerevisiae: Effect ofERG9 repression on sesquiterpene biosynthesis. Biotechnol. Bioeng. 2008, 99, 666-677.

(12) Albertsen, L.; Chen, Y.; Bach, L. S.; et al. Diversion of Flux toward Sesquiterpene Production inSaccharomyces cerevisiaeby Fusion of Host and Heterologous Enzymes. Appl. Environ. Microbiol. 2011, 77, 1033-1040.

(13) Mitsui, R.; Nishikawa, R.; Yamada, R.; Matsumoto, T.; Ogino, $\mathrm{H}$. Construction of yeast producing patchoulol by global metabolic engineering strategy. Biotechnol. Bioeng. 2020, 117, 1348-1356.

(14) Henke, N.; Wichmann, J.; Baier, T.; et al. Patchoulol Production with Metabolically Engineered Corynebacterium glutamicum. Genes 2018, 9, 219.

(15) Ma, B.; Liu, M.; Li, Z.-H.; Tao, X.; Wei, D.-Z.; Wang, F.-Q. Significantly Enhanced Production of Patchoulol in Metabolically Engineered Saccharomyces cerevisiae. J. Agric. Food Chem. 2019, 67, $8590-8598$. 
(16) Wu, S.; Schalk, M.; Clark, A.; Miles, R. B.; Coates, R.; Chappell, $\mathrm{J}$. Redirection of cytosolic or plastidic isoprenoid precursors elevates terpene production in plants. Nat. Biotechnol. 2006, 24, 1441-1447.

(17) Vranová, E.; Coman, D.; Gruissem, W. Network analysis of the MVA and MEP pathways for isoprenoid synthesis. Annu. Rev. Plant Biol. 2013, 64, 665-700.

(18) Zhan, X.; Zhang, Y. H.; Chen, D. F.; Simonsen, H. T. Metabolic engineering of the moss Physcomitrella patens to produce the sesquiterpenoids patchoulol and $\alpha / \beta$-santalene. Front. Plant Sci. 2014, 5, 636.

(19) Shimamura, M. Marchantia polymorpha: Taxonomy, Phylogeny and Morphology of a Model System. Plant Cell Physiol. 2016, 57, 230-256.

(20) Tsuboyama, S.; Kodama, Y. AgarTrap: a simplified Agrobacterium-mediated transformation method for sporelings of the liverwort Marchantia polymorpha L. Plant Cell Physiol. 2014, 55, 229-236.

(21) Bowman, J. L.; Kohchi, T.; Yamato, K. T.; et al. Insights into Land Plant Evolution Garnered from the Marchantia polymorpha Genome. Cell 2017, 171, 287-304.

(22) Asakawa, Y.; Ludwiczuk, A. Chemical Constituents of Bryophytes: Structures and Biological Activity. J. Nat. Prod. 2018, 81, 641-660.

(23) Antoniewic, M. R. Methods and advances in metabolic flux analysis: a mini-review. J. Ind. Microbiol. Biotechnol. 2015, 2, 317-325.

(24) Sauret-Güeto, S.; Frangedakis, E.; Silvestri, L.; et al. Systematic Tools for Reprogramming Plant Gene Expression in a Simple Model, Marchantia polymorpha. ACS Synth. Biol. 2020, 9, 864-882.

(25) Dahl, R. H.; Zhang, F.; Alonso-Gutierrez, J.; et al. Engineering dynamic pathway regulation using stress-response promoters. Nat. Biotechnol. 2013, 31, 1039-1046.

(26) Barghetti, A.; Sjögren, L.; Floris, M.; Paredes, E. B.; Wenkel, S.; Brodersen, P. Heat-shock protein 40 is the key farnesylation target in meristem size control, abscisic acid signaling, and drought resistance. Genes Dev. 2017, 31, 2282-2295.

(27) Bagchi, S.; Rathee, P.; Jayaprakash, V.; Banerjee, S. Farnesyl Transferase Inhibitors as Potential Anticancer Agents. Mini Rev. Med. Chem. 2018, 18, 1611-1623.

(28) Bikkul, M. U.; Clements, C. S.; Godwin, L. S.; et al. Farnesyltransferase inhibitor and rapamycin correct aberrant genome organisation and decrease DNA damage respectively, in HutchinsonGilford progeria syndrome fibroblasts. Biogerontology 2018, 19, 579602.

(29) Northey, J. G.; Liang, S.; Jamshed, M.; et al. Farnesylation mediates brassinosteroid biosynthesis to regulate abscisic acid responses. Nat. Plants 2016, 2, 16114.

(30) Stenøien, H. K. Adaptive basis of codon usage in the haploid moss Physcomitrella patens. Heredity 2005, 94, 87-93.

(31) Hemmerlin, A.; Harwood, J. L.; Bach, T. J. A raison d'être for two distinct pathways in the early steps of plant isoprenoid biosynthesis? Prog. Lipid Res. 2012, 51, 95-148.

(32) Tetali, S. D. Terpenes and isoprenoids: a wealth of compounds for global use. Planta 2019, 249, 1-8.

(33) Sarrion-Perdigones, A.; Falconi, E. E.; Zandalinas, S. I.; et al. GoldenBraid: an iterative cloning system for standardized assembly of reusable genetic modules. PLoS One 2011, 6, No. e21622. 